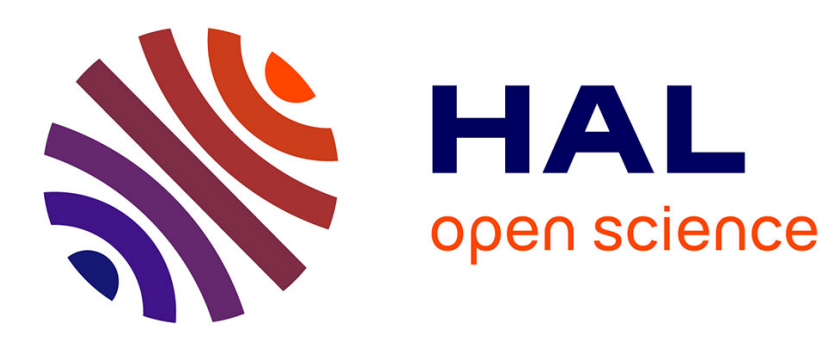

\title{
Guidelines for the Selection of FIFO Lanes and Supermarkets for Kanban-Based Pull Systems - When to Use a FIFO and When to Use a Supermarket
}

Christoph Roser, Masaru Nakano

\section{- To cite this version:}

Christoph Roser, Masaru Nakano. Guidelines for the Selection of FIFO Lanes and Supermarkets for Kanban-Based Pull Systems - When to Use a FIFO and When to Use a Supermarket. IFIP International Conference on Advances in Production Management Systems (APMS), Sep 2015, Tokyo, Japan. pp.282-289, 10.1007/978-3-319-22759-7_33 . hal-01431107

\author{
HAL Id: hal-01431107 \\ https://hal.inria.fr/hal-01431107
}

Submitted on 10 Jan 2017

HAL is a multi-disciplinary open access archive for the deposit and dissemination of scientific research documents, whether they are published or not. The documents may come from teaching and research institutions in France or abroad, or from public or private research centers.
L'archive ouverte pluridisciplinaire HAL, est destinée au dépôt et à la diffusion de documents scientifiques de niveau recherche, publiés ou non, émanant des établissements d'enseignement et de recherche français ou étrangers, des laboratoires publics ou privés.

\section{(c)(1)}

Distributed under a Creative Commons Attribution| 4.0 International License 


\title{
Guidelines for the Selection of FIFO Lanes and Supermarkets for Kanban-Based Pull Systems - When to Use a FIFO and When to Use a Supermarket
}

\author{
Christoph Roser ${ }^{1}$, Masaru Nakano ${ }^{2}$ \\ ${ }^{1}$ Karlsruhe University of Applied Sciences, Karlsruhe, Germany \\ christoph.roser@hochschule-karlsruhe.de \\ ${ }^{2}$ Graduate School of System Design and Management, Keio University, Yokohama, Japan \\ m.nakano@sdm.keio.ac.jp
}

\begin{abstract}
Modern lean production systems most often use a pull-based production control, usually implemented as kanban or CONWIP. The material flow in pull systems is managed using both FIFO lanes and supermarkets as inventories, which are well described in academic literature. However, there is so far little guidance as for when to use a supermarket and when to use a FIFO lane. This paper aims to provide an exhaustive list of considerations regarding the selection of a supermarket or FIFO lane between processes. While the detailed decisions depend on the particularities of the system, a number of general recommendations and considerations can be made. To the best knowledge of the authors, no such effort has been undertaken before, and we believe that this summary of considerations is long overdue and of immense value for the practitioner.
\end{abstract}

Keywords: Pull, FIFO, Supermarket, Kanban, Value Stream Design, Material Flow, Information Flow

\section{Introduction}

One key to a lean manufacturing system is to control production through a pull system. The most well-known approach to create a pull system is through the use of kanban in a loop with supermarkets and FIFO lanes. This system was developed at Toyota in Japan after World War II [1] and was first presented in English by [2]. Before we proceed, we will briefly explain the function of FIFO lanes and supermarkets. For details we refer to the vast body of literature on kanban, FIFO lanes, and supermarkets, as for example [3]. Please note that kanban systems are ill equipped to handle high-variety production, in which case a CONWIP system may be more suitable.

\subsection{FIFO Lanes}

FIFO stands for "First in - First Out." The first part that goes in the inventory is also the first part that goes out. There is a limit to the maximum number of parts 
in a FIFO lane. A full FIFO eventually stops the preceding process, whereas an empty FIFO naturally stops the succeeding process. The example system shown in Fig. 1 shows four processes connected through three FIFO lines. Please note that we will use the widely accepted value stream mapping nomenclature to describe value streams throughout this paper [4].

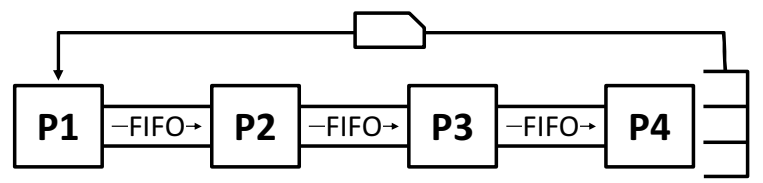

Fig. 1. Example of a four process kanban system with FIFO lanes and supermarkets using standard value stream mapping nomenclature

Only the first process P1 needs to receive separate information about the production plan - here in the form of a kanban - whereas all other processes simply process the next part in the FIFO lane. FIFO lanes also work very well with any type of product mix or lot size. However, it requires an identical material flow for all parts and thus is usually ill-suited for job shops; hence this paper focuses on flow shop type of production.

\subsection{Supermarkets}

Supermarkets can be seen as a number of FIFO lanes in parallel, one for each part type produced in the system. Hence the material flow is very similar to FIFO lanes, except that the flow is split by part type. However, the information flow is more complex in supermarkets. Whenever a part is removed from the supermarket, the information about the removed part has to travel back to the beginning of the loop to initiate the replenishment of this particular part type. This information is usually called kanban. The example of Fig. 1 shows one kanban loop with four processes. Operating the supermarket is slightly more complex than a simple FIFO lane, as the material flow and the information flow splits. Additionally, a supermarket usually takes more time to set up and implement compared to a FIFO lane. Finally, the number of kanban has to be determined and updated regularly, and the system has to be checked for lost kanban.

\subsection{Problem Statement: Options between FIFO Lanes and Supermarkets}

A manufacturing system with multiple processes has different options for kanban system implementations. Fig. 2 shows the kanban loop options for a manufacturing system with three processes. In option a), a single kanban loop covers all three processes. Option b) and c) use two kanban loops to implement a pull system for the three processes. Finally, option d) establishes a separate kanban loop for each process. The question this paper aims to address is: Which of these options is superior to the others for a specific production system? I would like to emphasize that all four options, a) to d), are usually completely valid pull systems. In general, the relation between number of possible system options $p$ and the number of separate processes $n$ 
is $p=2^{n-1}$. Between every sequential pair of processes there is the option to use either a FIFO lane as part of a bigger loop or to split it into two loops using a supermarket. The question is: When should a supermarket be used, and when a FIFO lane? Within this paper we assume that all materials are stored within a FIFO lane or a supermarket as part of a kanban based pull system.

a)

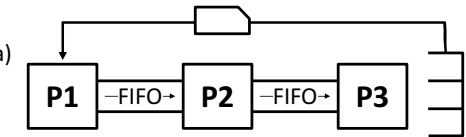

b)

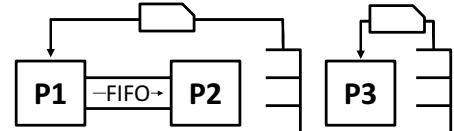

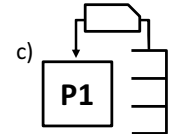

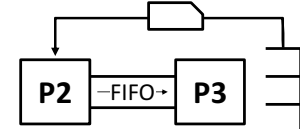

d)

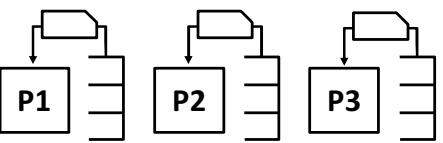

Fig. 2. Four options for a three-process system with FIFO lanes and supermarkets

\section{On the Placement of FIFO Lanes and Supermarkets}

\subsection{Use FIFO Whenever There Is No Particular Reason to Use a Supermarket}

Generally speaking, FIFO lanes have significantly less management overhead compared to supermarkets. Hence, the first guideline is to use a FIFO whenever there are no particular circumstances that would advise the use of a supermarket. This will also marginally improve the delivery performance for the same inventory, although the difference is not very large [5].

\subsection{Consider a Supermarkets for Production Lot Size Differences}

Some processes in the production system may have a different lot size from others. Assume you have a kanban loop with two processes and one FIFO lane in between as shown in Fig. 3. Depending on the lot sizes of the two processes P1 and $\mathrm{P} 2$, the system may come to a gridlock due to a lot size mismatch. Below is a complete list of possible situations with respect to lot size and their effect on the use of a FIFO lane.

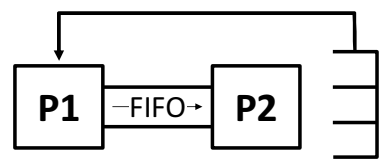

Fig. 3. Kanban loop with two processes and one FIFO lane.

a. If the processes have equal lot sizes, then no supermarket is needed.

b. If the process with the smaller lot size can accept any lot size equal to or larger than the other process, then there is no supermarket needed as long as the larger lot size is applied to the entire loop. For example, if the lot size of P1 is 100 parts, and 
the lot size of P2 is anything larger than 30 parts, then no supermarket is needed between the processes

c. If the process with the smaller lot size requires an exact lot size, then no supermarket is needed if and only if the larger lot size is an even multiple of the smaller lot size. For example, if the lot size of P1 is 100 parts, and the lot size of P2 is 50 parts, then no supermarket is needed between the processes since $\mathrm{P} 2$ can simply do two lots of exactly 50 parts each. Otherwise a supermarket is needed to decouple the processes and re-arrange the lot sizes for the different product types.

Hence, with different lot sizes there may be a situation where a supermarket is required for the system to function. Of course, in practical experience the lot size is rarely fixed, and the lot sizes of the system can be almost always adjusted to avoid the need for a supermarket as outlined in situation c) above.

\subsection{Consider a Supermarket before Creating Product Variants}

Another instance where a supermarket may be superior compared to a FIFO lane is during the creation of variants. Using again the example of Fig. 3, let's assume that the first process P1 has one variant only. During the second process, ten different variants are created based on the single product created by P1. Using a FIFO lane before creating variants has a number of disadvantages. The first and less important reason is that the information regarding the variant has to be included on the kanban card already on process P1. Yet, process P1 does not need this information. Hence, there is an unneeded information transport. Additionally, since P1 does not need this information, there is a possibility that this information is considered unimportant by $\mathrm{P} 1$, resulting in a higher likelihood of the information being lost.

The second and more significant reason is that the replenishment time becomes longer and the system more sluggish, since a kanban has to pass through all processes in the longer loop, which takes more time. All these problems can be avoided through the use of a supermarket. P1 would produce only generic electronics for the clock, while P2 would assemble it in the color on the highest-priority kanban card waiting for production.

\subsection{Consider a Supermarket after the Final Process and Before the Customer}

a)

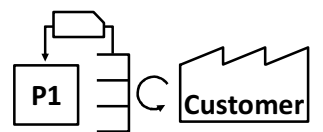

b)

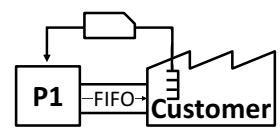

Fig. 4. Kanban loop excluding and including the customer

It is common practice in kanban systems to have a supermarket before your customer. It is theoretically possible to include the customer in the loop as shown in option b) in Fig. 4. However, this requires close interaction and cooperation with the customer. While it is possible, it requires additional effort and care to make sure all kanban cards eventually return to the producer for replenishment. Much more com- 
mon and usually much easier to implement is to have a supermarket before the customer as shown in option a) below. Since the kanban never leaves the producer's sphere of influence, it is much easier for the producer to create a smooth pull production.

\subsection{Consider a Supermarket for Merging Material Flows}

A supermarket may also be highly beneficial for merging material streams. Assume there are two processes, $\mathrm{P} 1$ and $\mathrm{P} 2$ that jointly feed a third process, $\mathrm{P} 3$. If the material flow between $\mathrm{P} 1 / \mathrm{P} 2$ and $\mathrm{P} 3$ uses FIFO lanes, a number of different problems may result, regardless whether there are two FIFO lanes as shown in a) or one FIFO lane as shown in b) in Fig. 5. This is also regardless whether process P3 merges the two parts from P1 and P2 through assembly or merely processes them individually, although in the reasoning we need to distinguish these cases.
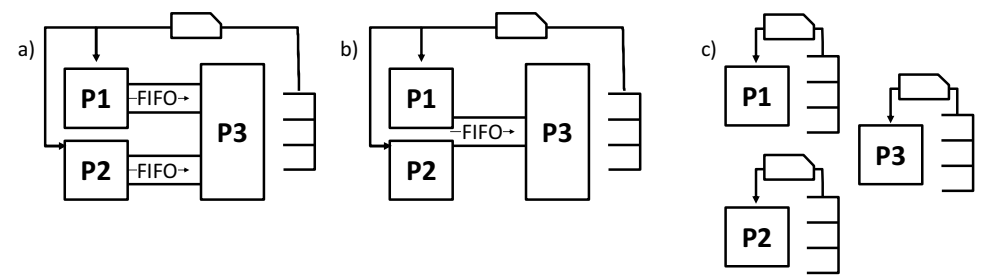

Fig. 5. Merging material flows

If $\mathrm{P} 3$ assembles products and requires parts from both $\mathrm{P} 1$ and $\mathrm{P} 2$ to work, the kanban has to reach both processes. While an electronic kanban can easily be sent to two processes, this is more difficult for physical kanban like paper or boxes. These kanban also somehow have to merge again in P3, resulting in two kanban per unit in the supermarket. Overall, it will make production control more demanding and will create additional potential for errors. Furthermore, if there are variants, the parts coming from $\mathrm{P} 1$ and $\mathrm{P} 2$ have to match the desired product. If for any reason the product sequence in either P1 or P2 becomes out of order, P3 may come to a gridlock if the two provided parts do not match. This gridlock then has to be resolved manually.

If $\mathrm{P} 3$ does not assemble products but merely processes one of the products, the kanban card would go to either P1 or P2. Hence additional decision making is needed to decide which process the kanban card goes to. If the processes P1 and P2 would differ the situation would become even more complex. Additionally, at P3 in example a), another decision is needed regarding which FIFO lane to process first. This is less of a problem in b), although production lot sizes and sequences from P1 and P2 become out of order. Hence merging material flows may benefit from the use of additional supermarkets.

\subsection{Consider a Supermarket for Dividing Material Flows}

Similar to merging material flows, dividing material flows may also benefit from supermarkets. A FIFO lane as shown in a) or b) in Fig. 6 will require additional 
decision making to determine if the produced part goes to P2 or P3 in both situation a) and b). Furthermore, especially in case b), there may be the possibility that either process $\mathrm{P} 2$ or $\mathrm{P} 3$ has to wait since the foremost part in the FIFO is intended for the other process. While overall this is less complicated than a merging material flow, depending on the circumstances a supermarket may also be beneficial for dividing material flows.

a)

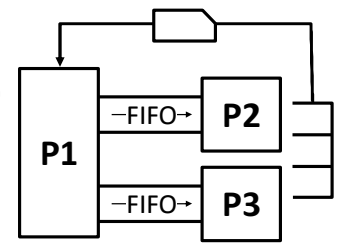

b)

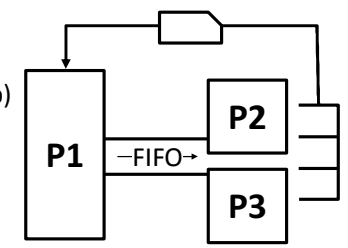

c)

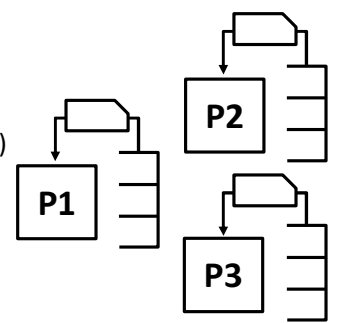

Fig. 6. Dividing material flows

Splitting material flows in most discrete manufacturing represent a process P1 that alternatingly provides for process P2 and P3. However, in the rare situations where process $\mathrm{P} 1$ actually disassembles one product into two subcomponents, there is a similar problem with the splitting of kanban as described with the merging material flows. In any case, any division in material flow may be a candidate for closer consideration for a supermarket.

\subsection{Consider a Supermarket for Very Different Speeds or Times}

Yet another instance where a supermarket may be beneficial is for time differences in production. Such time differences may come from significantly different cycle times, changeover times, or shift patterns. The problem is small if the time difference is small, but may become larger depending on the time difference. Assume again in Fig. 3 that P1 and P2 have very different speeds or working times. Standard practice is to decouple these processes with buffer stock.

Such buffer inventory could, for a pull system, consist of either a FIFO lane or a supermarket, with similar inventory levels. However, if the system lacks robustness and is sensitive to changing customer demands, a FIFO lane may have a disadvantage due to the time delay. The production sequence of the faster process would be defined long beforehand. While this poses no problem in theory, in practice production systems are rarely static. Within the duration of a shift, priorities can change and material can become available or (more likely) missing due to delivery delays, quality problems, or other mishaps. In any case the ideal production sequence for subsequent processes may change while the parts are in the inventory. A supermarket allows for an easier access of the highest-priority materials compared to a FIFO lane. Therefore, the longer the time differences and therefore the buffer inventory reach, the higher the benefit of a supermarket instead of a FIFO lane. 


\subsection{Consider a Supermarket for Large Distances between Processes}

The above example of long delays between creating the production pattern in the preceding process and using the pattern in the succeeding process also applies for transport delays. Taking an extreme example, a shipment of goods from Shanghai to San Francisco can easily take two weeks or more, not even including delays by customs or through unloading. While in this example it is already nearly impossible to maintain FIFO physically, even a data-based FIFO where each part is delivered in the sequence it was loaded is difficult. However, more significant is again the time delay. As described above, circumstances and priorities may change significantly while the goods are in shipping. This change of circumstances would then require a break in the FIFO sequence. This would be much easier with a supermarket on one or even both sides. While ideally maintaining a product-specific FIFO sequence, the receiving side can prioritize the production much easier with a supermarket than using a FIFO lane. Therefore, it may be beneficial to split a larger loop into two parts by adding a supermarket if the physical or time-wise distance between processes becomes too large. This is definitely true for different continents, but also applies to different locations within one continent or country, and may sometimes even be sensible for different locations within one plant.

\subsection{Consider a Supermarket for High Demands on Agility}

Long replenishment times in one kanban loop can be shortened by splitting it up into two separate loops. Hence, adding more loops may make the production system more agile and responsive to changes in demand. Ideally, these changes can come from the customer side, but in less-than-perfect systems they may also arise due to problems elsewhere including material availability, machine troubles, or availability of skilled labor. In any case, it may be necessary to change the production plan to match the new circumstances. If there is only one long kanban loop, any change in the production plan has to go through the entire value stream before resulting in a finished product at the end. Smaller loops will be able to react much quicker. Hence, having multiple loops will allow each loop to become more agile and to react faster to changes. If the changes stem only from the customer side, a small loop at the end will suffice. However, if the changes are also caused by other factors along the value stream, splitting the entire value stream into multiple loops by adding supermarkets may help.

\subsection{Consider a Supermarket for Changes in Responsibility}

All of the above considerations for using a supermarket were based on logical reasons related to time and quantity in order to get the maximum effect with a minimal effort. This last reason, however, is different. It is not based on logical conclusions of cause and effect, but rather on the intricate behavior of the human mind. Ideally, if there are problems, the goal of the people involved should be to resolve the problem as fast as possible and resume operations. Practically, depending on the company culture, the people may be more concerned with finding the responsible party than with solving the problem (colloquially known as the blame game). There is 
often disagreement on whom to blame, and, naturally speaking, each person involved considers her- or himself innocent and sees the problem with someone else. Far too many companies extend a greater effort on this blame game than on fixing the problem. While a mere supermarket cannot eliminate this problem, it can reduce its spread as a sort of separator of responsibilities. The supplying party should keep the material stocked, and the demanding party should not exceed certain limits on fluctuation. Hence a supermarket at the boundaries of changes in responsibility may be able to contain the overhead friction of problems within one area of responsibilities.

\section{Conclusion}

While generally speaking a FIFO lane is much easier to manage and hence preferable in the often-hectic environment of the modern shop floor, there are a number of reasons when it may be beneficial to split a larger kanban-based pull loop into smaller loops through the adding of a supermarket. All of the reasons can also be managed through a FIFO lane. However, this may require additional efforts and nonstandard procedures as, for example, breaking the FIFO sequence of parts. Besides the additional manual effort, a possible break in the FIFO sequence can lead to higher fluctuations in throughput times and production delays for some products and hence missed deadlines. Furthermore, this can also result in aging of products and possibly higher inventories and scrap rates. Depending on the circumstances, a supermarket may be the better tool for inventory management in many of the above cases. Of course, a vast number of additional circumstances will also influence this decision on the shop floor. However, the above paper will help and guide the practitioner in designing a value stream by listing the possible considerations for a supermarket in contrast to the standard solution of a FIFO lane. This will eliminate waste and create a smoother, more productive manufacturing system.

\section{Bibliography}

[1] T. Ohno, Toyota Production System: Beyond Large-scale Production, 1st Edition. Productivity Press, 1988.

[2] Y. Sugimori, K. Kusunoki, F. Cho, and S. Uchikawa, "Toyota production system and Kanban system Materialization of just-in-time and respect-for-human system," Int. J. Prod. Res., vol. 15, no. 6, pp. 553-564, Jan. 1977.

[3] Y. Monden, Toyota Production System - An Integrated Approach to Just-In-Time. Engineering \& Management Pre, 1998.

[4] M. Rother and J. Shook, Learning to See: Value-Stream Mapping to Create Value and Eliminate Muda: Value Stream Mapping to Add Value and Eliminate Muda. Lean Enterprise Institute, 1999.

[5] D. Wiesse, "Analyse des Umlaufbestandes von Verbrauchssteuerungen in Abhängigkeit von der Nutzung von Supermärkten und FiFo-Strecken," Karlsruhe University of Applied Sciences, Karlsruhe, Germany, 2015. 